\title{
Diamond-Graphene Composite Nanostructures
}

\author{
Péter Németh, ${ }^{*}$ Kit McColl, $*$ Rachael L. Smith, Mara Murri, Laurence A. J. Garvie, Matteo Alvaro, \\ Béla Pécz, Adrian P. Jones, Furio Corà, Christoph G. Salzmann,* and Paul F. McMillan*
}

Cite This: https://dx.doi.org/10.1021/acs.nanolett.0c00556

Read Online

ACCESS

Wl Metrics \& More

Article Recommendations

Supporting Information

ABSTRACT: The search for new nanostructural topologies composed of elemental carbon is driven by technological opportunities as well as the need to understand the structure and evolution of carbon materials formed by planetary shock impact events and in laboratory syntheses. We describe two new families of diamondgraphene (diaphite) phases constructed from layered and bonded $\mathrm{sp}^{3}$ and $\mathrm{sp}^{2}$ nanostructural units and provide a framework for classifying the members of this new class of materials. The nanocomposite structures are identified within both natural impact diamonds and laboratory-shocked samples and possess diffraction features that have previously been assigned to lonsdaleite and postgraphite phases. The diaphite nanocomposites represent a new class of high-performance

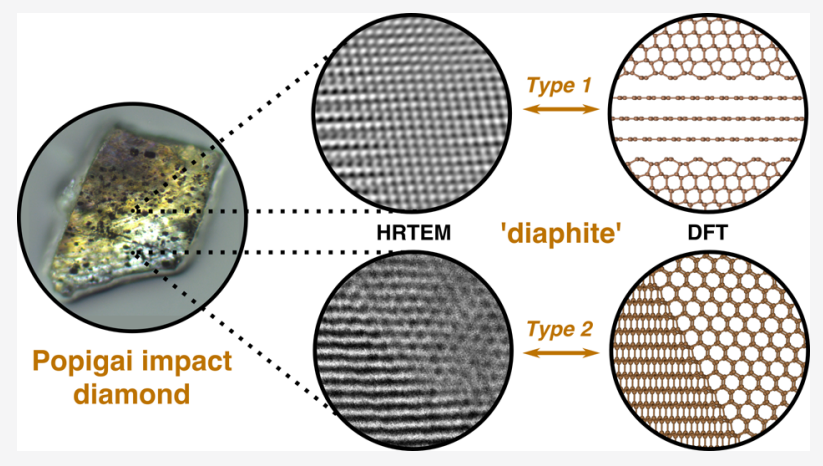
carbon materials that are predicted to combine the superhard qualities of diamond with high fracture toughness and ductility enabled by the graphitic units and the atomically defined interfaces between the $\mathrm{sp}^{3}$ - and $\mathrm{sp}^{2}$-bonded nanodomains.

KEYWORDS: Graphene-diamond nanocomposite, high-resolution TEM, density functional theory calculations, $s p^{2}$ - and $s p^{3}$-bonded nanomaterials, mechanical properties

Tnterest in new carbon allotropes is driven by the desire to identify novel materials for advanced technologies as well as to understand the role and fate of carbon phases in planetary processes. ${ }^{1}$ The stable form of carbon at ambient conditions is layered hexagonal graphite with $\mathrm{sp}^{2}$ bonding. ${ }^{2}$ The nanomaterial graphene, which has remarkable electronic and mechanical properties, consists of single or very few stacked layers of the graphite structure. ${ }^{3}$ Following highpressure (HP) and high-temperature (HT) treatment, graphite and graphitic carbon are transformed to $\mathrm{sp}^{3}$-bonded diamond that forms a superhard material with technological importance. This material has tetrahedrally bonded carbon atoms that are covalently linked to form six-membered rings in a "chair" conformation, and the atoms are stacked into layers according to a cubic (c) close-packing arrangement or the $3 \mathrm{C}$ stacking polytype. ${ }^{2}$ A metastable $\mathrm{sp}^{3}$-bonded carbon allotrope identified within natural impact diamonds and laboratory samples created under static HP and HT or shock conditions is "lonsdaleite", a dense crystalline form displaying diffraction features consistent with hexagonal symmetry. $^{4-12}$ Observations of such hexagonal diffraction features provide an important mineralogical marker for the $P$ and $T$ conditions recorded in diamonds from bolide impact sites. ${ }^{5,8,13}$ Lonsdaleite was interpreted as a $2 \mathrm{H}$ polytype with carbon layers stacked in a hexagonal fashion $(h)$, although recent analyses suggest that natural and experimentally produced lonsdaleite samples are more accurately described as nanotwinning and stacking-disordered sequences of $c$ and $h$ units. ${ }^{14-18}$ Density functional theory (DFT) calculations as well as experimental measurements suggest that the hardness and other mechanical properties of lonsdaleite may be superior to that of cubic diamond, ${ }^{19}$ thus motivating the search for identifying the $\mathrm{sp}^{3}$-bonded hexagonally stacked polytype among natural materials, or preparing it in the laboratory. ${ }^{20,21}$ Computational studies suggest a wide range of novel carbon polymorphs that might be produced in the laboratory or discovered in natural samples. ${ }^{22-24}$ In particular, several metastable carbon phases have been identified as being associated with the transition between $\mathrm{sp}^{2}$ - and $\mathrm{sp}^{3}$-bonded structures. $^{25-30}$

It was first shown by analysis of high-resolution transmission electron microscopy (HRTEM) images that hexagonal features appearing in the diffraction patterns of impact diamonds could be interpreted as cubic (3C) diamond containing a high density of stacking faults associated with nanoscale twinning. ${ }^{14}$ Analysis of the X-ray diffraction profiles

Received: February 9, 2020

Revised: $\quad$ March 20, 2020

Published: April 8, 2020 


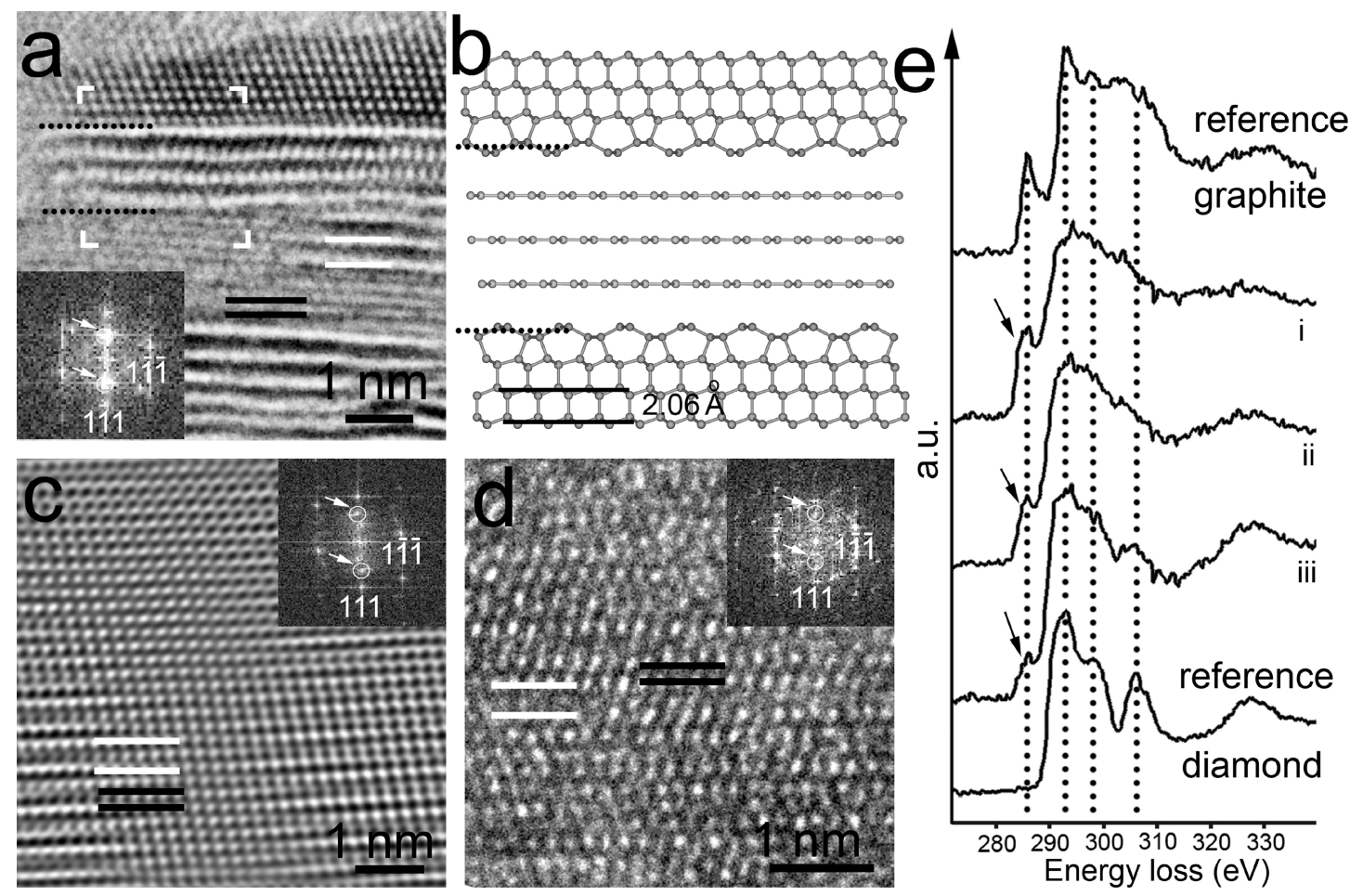

Figure 1. HRTEM images and EELS data showing the existence of type 1 diaphite nanocomposites resulting from few-layered graphene units inserted within $\{111\}$ diamond. (a) HRTEM image of a graphene-diamond particle from the shocked Gujba meteorite (adapted with permission from ref 27, Figure 5b. Copyright 2014 Mineralogical Society of America). White and black lines mark crystallographically related $\sim 3.4 \AA\{00 l\}$ graphene and $\sim 2.1 \AA\{111\}$ diamond spacings, respectively. The FFTs in the inset to this and other TEM images show the characteristic graphene (marked by white circles) and $\langle 011\rangle$ diamond reflections. (b) DFT-calculated structure model of type 1 diaphite for the area marked by white corners of $(\mathrm{a})$. The model shows the Pandey $(2 \times 1)$ reconstructed surfaces ${ }^{42}$ of the bottom and top $\{111\}$ diamond layers (marked by dotted lines), the arrangement of which is unresolved on the experimental image. (c) Background-filtered image (unprocessed image shown in Figure S1a) of an interfingering graphene-diamond nanocomposite observed within a Popigai diamond. (d) Overlapping graphene-diamond nanocomposites from a 1.5 Mbar laboratory-shocked graphite sample show continuous $2.06 \AA$ (diamond) and $3.4 \AA$ (graphene) fringes. Although the superposition of graphene and diamond units of type 1 diaphite makes the exact determination of individual components challenging, the FFT shows the characteristic diffraction features of type 1 diaphite. (e) EELS data for selected graphene-diamond nanocomposite regions (shown in Figure S2) from a Popigai diamond indicate mixed $\mathrm{sp}^{2}$ (graphitic) and $\mathrm{sp}^{3}$ (diamond) bonding.

of natural and laboratory-shocked diamond samples using the MCDIFFaX modeling technique later correlated the appearance of lonsdaleite features with different proportions of $h$ vs $c$ layer stacking within diamond polytype structures. ${ }^{16,17,31}$ The average degree of hexagonality $\left(\Phi_{h}\right)$ and stacking disorder among the $c$ and $h$ layers can be represented on a stackogram plot. ${ }^{16,17,31}$ These analyses were based on the assumption that the structures contained only $\mathrm{sp}^{3}$-bonded carbon atoms.

Introducing hybrid materials with $\mathrm{sp}^{2}$ - and $\mathrm{sp}^{3}$-bonding give rise to additional features into the images and diffraction patterns. From HRTEM studies of an extraterrestrially shocked meteorite, Garvie et al. ${ }^{27}$ described crystallites composed of few-layered graphene domains embedded within and exhibiting a topotactic relationship to slabs of diamond. Mixed $\mathrm{sp}^{2}$ - and $\mathrm{sp}^{3}$ - bonding is also observed in amorphous carbon forms with important technological applications. ${ }^{32}$ For example, amorphous diamond-like carbons exhibiting a high degree of tetrahedral bonding contained within an $\mathrm{sp}^{2}$-bonded graphitic matrix can achieve hardness values approaching that of diamond, ${ }^{33}$ while "hard carbon" materials with a similar nanocomposite structure are being developed for their reversible $\mathrm{Na}$-ion intercalation properties as battery anodes. ${ }^{34}$ A two-dimensional (2D) diamond-graphene crystalline nanostructure termed "diaphite" was proposed to form following photoexcitation of graphite. ${ }^{35,36}$ However, the existence of extended diaphite nanostructures within the $\mathrm{sp}^{3}$ bonded matrix of diamond has not been discussed.

Here we show evidence for two families of $\mathrm{sp}^{3}-/ \mathrm{sp}^{2}$-bonded carbon nanostructures that form the basis for a new class of crystalline nanocomposites that we call diaphite allotropes. We find that these structures occur within natural as well as laboratory-shocked samples and that features of their diffraction signatures match those previously reported for lonsdaleite as well as the postgraphite phases including $\mathrm{M}$ carbon, ${ }^{7,37-40}$ thus motivating a reappraisal of previous reports of these materials. Despite its high compressive and tensile strength, diamond exhibits a low fracture resistance as a result of crack propagation along cleavage planes. However, inclusion of diaphite nanocomposite structures would allow the material to absorb or deflect incipient crack formation, thus increasing its fracture resistance. ${ }^{41}$

HRTEM images of impact diamonds and laboratoryshocked graphite reveal the intrinsic structural complexity of these crystalline carbon materials. Nanostructures that exhibit lattice fringe spacings of both $\sim 3.4 \AA$ (closely matching the $\{001\}$ reflection of graphite) and $\sim 2.1 \AA$ (consistent with both $\{111\}$ diamond and $\{100\}$ graphite) have been observed in both natural and synthetic samples (Figures 1, 2, and S1S3). However, instead of suggesting the presence of independent graphite, lonsdaleite, or diamond within the 

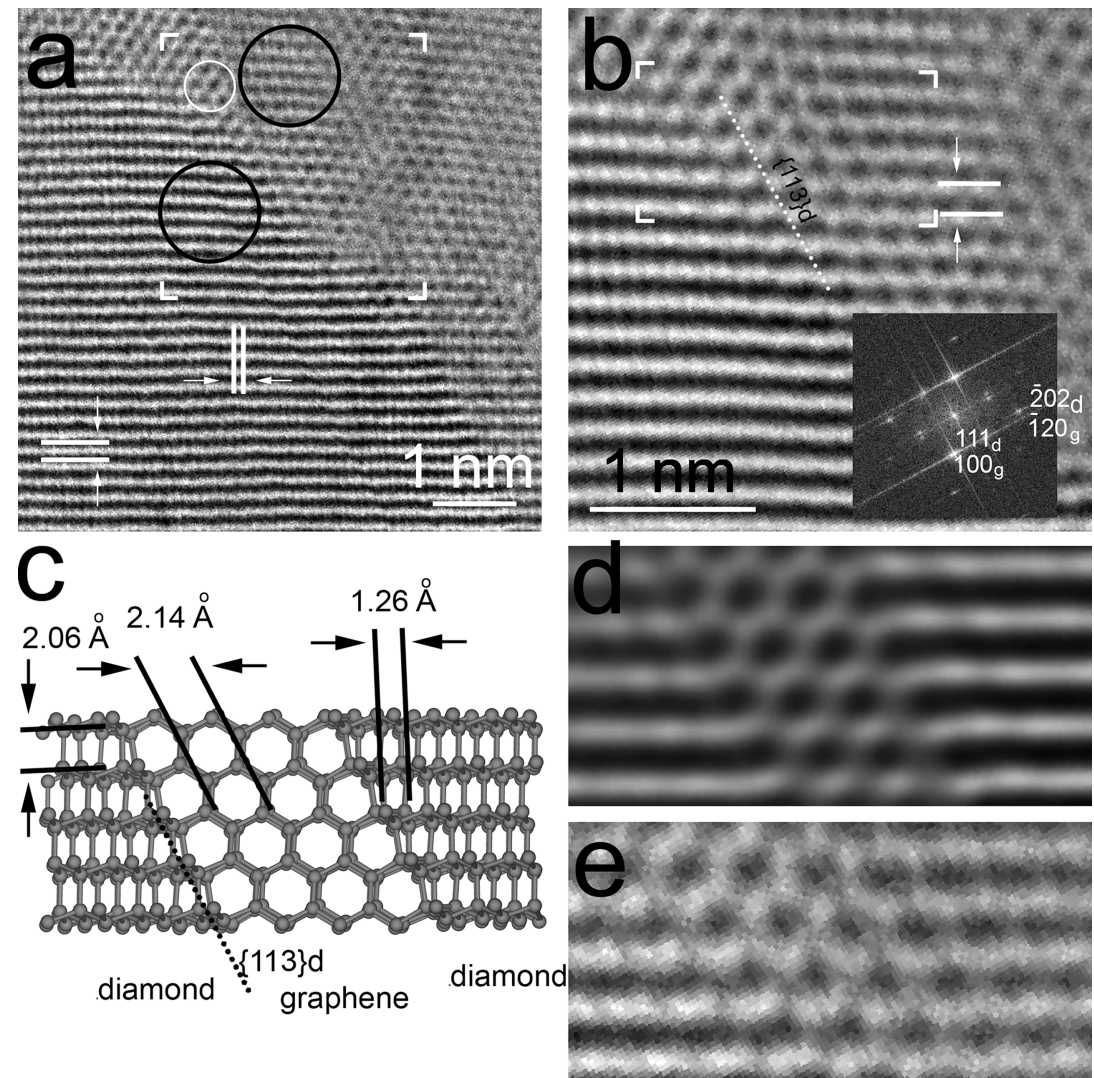

Figure 2. HRTEM images and DFT modeling of a type 2 diaphite nanocomposite containing graphitic layers inserted at high angles within $\{113\}$ diamond. (a) HRTEM image from a Popigai diamond shows perpendicular 2.1 and $1.26 \AA$ (contoured by a black circle) as well as hexagonally arranged 2.1 A fringes (contoured by a white circle). (b) Background filtered image calculated from the area marked by white corners of (a). The FFT in the inset shows $\langle 001\rangle$ graphene $h k l_{\mathrm{g}}$ partly overlapping with $\langle 121\rangle$ diamond $h k l_{\mathrm{d}}$ reflections. (c) Structure model of type 2 diaphite and its characteristic $d$ spacings obtained from DFT calculations. (d) The simulated HRTEM image calculated from the structure shown in (c) using the experimental microscopy conditions successfully reproduces the image contrast of the observed features (e) from the area marked by white corners of $(b)$.

samples, ${ }^{10,14}$ the $\sim 3.4 \AA$ fringes appear as domains consisting of few-layered graphene to graphitic domains contiguous with the $\{111\}$ diamond layers (Figures 1, S1, and S2). Their lateral extent normal to the layer stacking direction ranges up to a few nanometers, and they terminate within the $\mathrm{sp}^{3}$ bonded lattice. We refer to this as a type 1 diaphite nanostructure.

A TEM image of diaphite is a $2 \mathrm{D}$ projection of superimposed nanodomains. As a result, the graphitic and diamond regions are observed most clearly in thin $(<20 \mathrm{~nm})$ sections (Figure 1a). The DFT structure model of few-layered graphene sandwiched between $\{111\}$ diamond slabs (Figure 1b) not only reproduces the image contrasts consistent with type 1 diaphite but also reveals the interface corresponding to the Pandey $(2 \times 1)$ reconstructed surface. ${ }^{42}$ In thicker $(<50$ $\mathrm{nm})$ samples, the graphene and $\{111\}$ diamond fringes are less visible, but their contributions can be detected in fast Fourier transforms (FFT) of the HRTEM data (Figures 1c,d and S1). Our electron energy-loss (EELS) spectra show the presence of both $\mathrm{sp}^{2}$ - and $\mathrm{sp}^{3}$-bonded carbon coexisting within the type 1 diaphite materials (Figure 1e). The peak near 285 $\mathrm{eV}$ represents electronic transitions from $1 \mathrm{~s}$ core states to unoccupied $\pi^{*}\left(2 \mathrm{p}_{z}\right)$ states of $\mathrm{sp}^{2}$-bonded carbon. This peak is most intense in those regions where graphitic domains are clearly visible in the HRTEM images (Figure S2).
A second diaphite nanostructure, which we call type 2 diaphite, consists of hexagonally arranged graphitic carbon layers inserted within and bonded at high angles to the $\mathrm{sp}^{3}$ bonded diamond surfaces (Figures 2 and S3). HRTEM images of this diaphite are characterized by $\langle 121\rangle$ diamond domains and subnanometer-sized regions containing $\sim 2.1 \AA$ fringes arranged in a hexagonal pattern (Figures 2a,b and S3). Both the HRTEM images and the FFT indicate a topotaxial relationship between the two domain types (Figure $2 \mathrm{~b}$ ). A similar image from the Canyon Diablo meteorite had previously been interpreted as two- and four-layer-thick \{113\} diamond twins. ${ }^{14}$ However, our DFT calculations described below and further comparison with the simulated HRTEM images indicate that this nanostructure corresponds to a nanocomposite consisting of $\mathrm{sp}^{2}$ - and $\mathrm{sp}^{3}$-bonded carbon regions (Figure $2 \mathrm{c}-\mathrm{e}$ ).

We performed DFT calculations on atomic models of type 1 and 2 diaphite structures (see Supporting Information for details) to reveal the structural relationships between the $\mathrm{sp}^{2}$ and $\mathrm{sp}^{3}$-bonded nanodomains and the stability of our diaphite structures relative to other carbon allotropes (Figure 3). In both structure types, the models were constructed to include varying amounts of diamond $(d)$ vs graphene $(g)$ content (Figure 3a,b). The relative proportion of interface regions between the two structural units was varied by modifying the size of the unit cell, with increasingly large cells producing a 


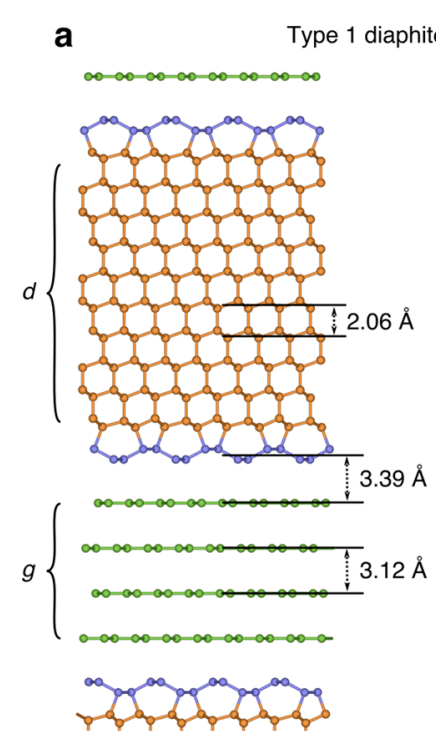

$(g=4, d=9)$
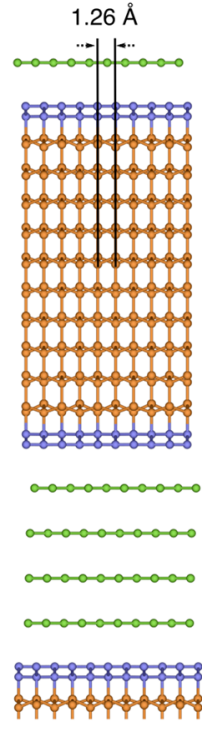

side view

c

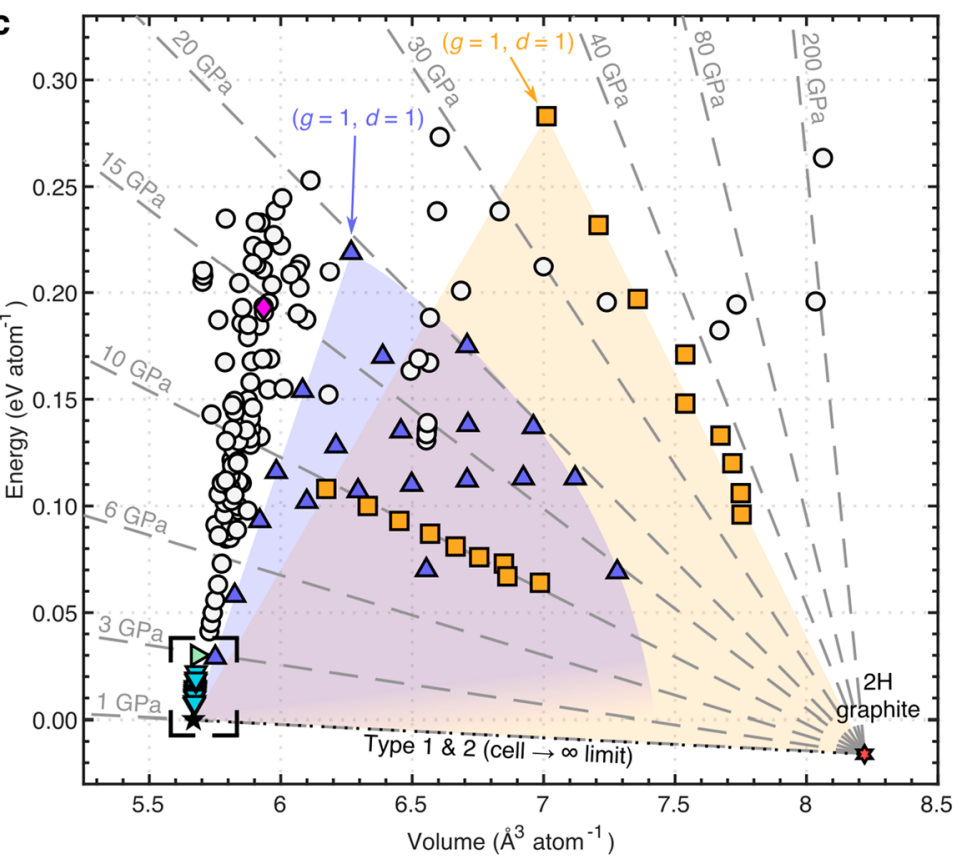

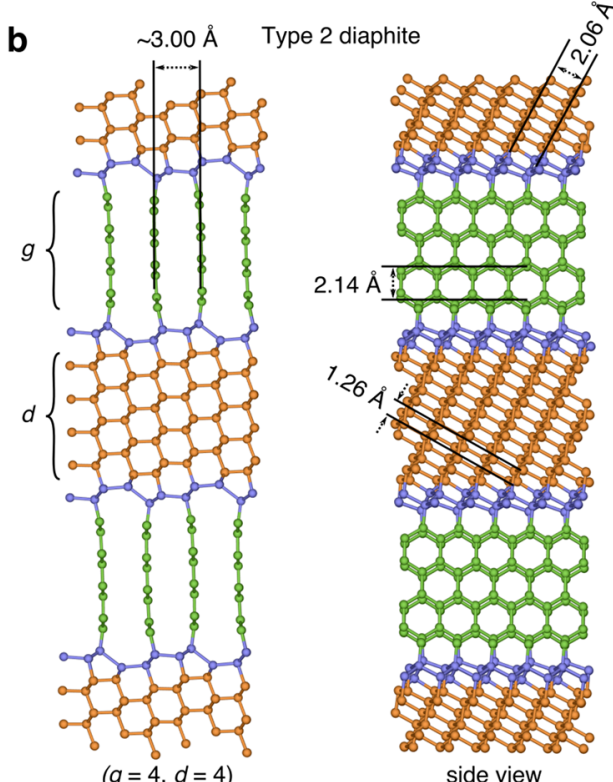

side view
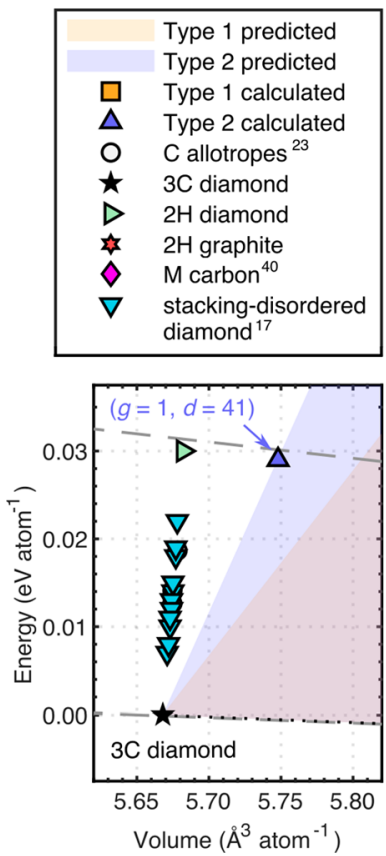

Figure 3. Structural examples of type 1 and 2 diaphite structures and a comparison of DFT calculated energy $(E)-$ volume $(V)$ values for diaphite structures and a range of stable and metastable carbon allotropes. Model structures for the basic type 1 diaphite (a) and type 2 diaphite (b). Their structures are defined in terms of the number of diamond $(d)$ and graphite $(g)$ components. In type $1, d$ defines the number of $\{111\}$ diamond layers between Pandey $(2 \times 1)$ reconstructed surfaces, ${ }^{42}$ and $g$ is the number of $\{001\}$ graphite layers. Type 1 models can be constructed with $d$ and $g$ independently taking any number between $1 \rightarrow \infty$. In type $2, d$ is the number of $\{113\}$ diamond layers, and $g$ is the number of $\{100\}$ graphite layers between the diamond regions. The constraints imposed by the choice of unit cells require that for type 2 models $d+g=2 n$, where $n$ is an integer. For both structures, $g=1, d=1$ results in the highest density of interfaces. Structure examples shown are $g=4, d=9$ (type 1 ) and $g=5, d=5$ (type 2). Green, orange, and blue units correspond to $\mathrm{sp}^{2}$-bonded graphene, $\mathrm{sp}^{3}$-bonded diamond, and $\mathrm{sp}^{2}$ - and $\mathrm{sp}^{3}$-bonded atoms in Pandey $(2 \times 1)$ reconstruction, respectively. (c) $E-V$ plot of type 1 and 2 diaphite and other carbon allotropes. Markers indicate calculated structures. The predicted $E-V$ regions in which type 1 and 2 diaphite structures can appear under ambient conditions are indicated by the shaded regions. Dashed lines indicate estimated pressures as the $E(V)$ slopes that can be attained in static or dynamic compression experiments or shock impacts. The panel in the bottom right expands the region indicated in black brackets in (c). The labels for calculated type 1 and 2 diaphite structures are shown in Figures S5 and S8.

lower density of interfaces. The two types of diaphite nanostructures give rise to the range of image and diffraction features observed experimentally in natural and laboratoryshocked samples (Figures 1, 2, and S1-S3).

One striking aspect of the model structures is the significantly smaller interlayer distances observed for the few-layered graphene domains within type 2 diaphites $(\sim 3 \AA)$. This spacing indicates that the graphene layers are compressed within the nanocomposite structure (Figure $3 \mathrm{~b}$ ), and their bonding, stacking, and electronic properties may be altered from those of bulk graphite or graphene. Although a similar interlayer contraction appears to be predicted for type 
1 diaphite (Figure 3a), this arises since the dispersion correction used in our DFT calculations overestimates the attractive forces between $\mathrm{sp}^{2}$ layers. However, that phenomenon does not affect the type 2 structures. The $\{100\}$ spacings of the graphene layers $(2.14 \AA)$ in type 2 diaphites are also expanded relative to those of free-standing graphene $(2.12 \AA)$. This lattice spacing closely matches the $\{100\}$ reflection $(2.18$ A) that has been assigned to lonsdaleite. ${ }^{4-13}$

The relative energies of type 1 and 2 diaphite nanostructures were plotted on an energy $(E)$-volume $(V)$ chart (Figure 3c) and compared with $2 \mathrm{H}$ graphite, cubic (3C) and hexagonal $(2 \mathrm{H})$ diamond, a range of $\left(\begin{array}{llll}\ldots & c_{n} h_{m} & \ldots\end{array}\right)$ stacking disordered diamond polytypes, and $>200$ other metastable carbon structures reported in the SACADA database. ${ }^{23}$ The diagram also plots estimated pressures as the $E(V)$ slopes that might be attained in static or dynamic compression experiments, including those encountered during planetary bolide impact events. As the relative proportions of $\mathrm{sp}^{2}$ - and $\mathrm{sp}^{3}$ bonded domains within the diaphite model structures are varied, the volume evolves systematically between the locus of points for $3 \mathrm{C}$ to $2 \mathrm{H}$ diamond polytypes and $2 \mathrm{H}$ to $3 \mathrm{R}$ graphite. The energy can be varied both by changing the relative contribution from each domain and by increasing the size of the unit cell, which has the effect of lowering the density of interface regions resulting in a lower overall energy. Extrapolating calculated results for type 1 and 2 structures with fixed $d$ or $g$ components toward infinitely large cells (i.e., $d=1, g=1,2,3, \ldots \rightarrow \infty)$, allows the prediction of the $E-V$ limits in which the different structures may be produced under equilibrium conditions (Figures S5 and S8). In the limit of $d=$ 1 and $g=\infty$, the type 1 structures attain the same energy and volume as $2 \mathrm{H}$ graphite. However, our type 2 structures always have a lower volume than $2 \mathrm{H}$ graphite due to the contracted interlayer distance between graphitic planes, and the locus of points for $d=n, g=\infty$ structures does not extrapolate to bulk $2 \mathrm{H}$ graphite. Our models are constrained by size and threedimensional periodicity, so different structural behavior might be observed in real materials.

The range of type 1 diaphite structures depicted as orange squares in Figure $3 c$ spans a locus of points that could be accessed by compression of graphite to between approximately 10 and $40 \mathrm{GPa}$, neglecting finite temperature effects and activation energy barriers. Large cells containing low densities of interfaces can achieve energy values that are competitive with the transition pressures from $2 \mathrm{H}$ graphite to diamond polytypes with a high degree of hexagonality (Figure $3 c)$. We demonstrate this by calculating the type $2(g=1, d=$ 41) structure (Figures S5-S8), which has a lower energy (in $\mathrm{eV}$ atom ${ }^{-1}$ ) than $2 \mathrm{H}$ diamond and falls below the transition pressure from $2 \mathrm{H}$ graphite to $2 \mathrm{H}$ diamond (expanded region of Figure 3c). This plot reveals that the formation of diaphite nanocomposite structures could provide low-energy solutions existing between fully $\mathrm{sp}^{3}$ - or $\mathrm{sp}^{2}$-bonded cubic-hexagonal diamond and graphite phases, while maintaining fully saturated $\mathrm{C}-\mathrm{C}$ linkages. These diaphite nanostructures might also be encountered during recovery to ambient pressure of $\mathrm{sp}^{3}$-bonded cubic-hexagonal diamond polytypes or by heating metastable $\mathrm{sp}^{3}$ carbon phases at ambient pressure, as they return toward the thermodynamically stable graphitic phase. These structures might also form during diamond surface graphitization according to the $a b$ initio theoretical prediction of De Vita et al. ${ }^{43}$
The question arises as to whether the diaphite structures can be revealed from X-ray diffraction and Raman data. Figure 4a shows the diffraction patterns of several Popigai diamonds previously fitted using $h / c$ diamond stacking disorder models. ${ }^{17}$ This approach enabled us to reproduce the diffraction pattern including the diffuse diffraction features at $\sim 20$ and $35^{\circ} 2 \theta \mathrm{MoK} \alpha$. From these fits, hexagonality indices (i.e., percentages of hexagonal diamond stacking) were obtained (Figure 4a). The only diffraction features that were previously not included in our fits were the sharp, but fairly weak peaks at $\sim 12^{\circ} 2 \theta \mathrm{MoK} \alpha$, which were observed for the two samples with highest hexagonal index. These peaks are commonly attributed to the $00 l$ peaks of graphite. To test if this peak and perhaps the other diffuse features could arise from diaphite structures, we extended our DIFFaX model to contain type 1 diaphite structures, which were found to be most abundant in our samples. This new DIFFaX model includes $c / h$ diamond stacking, the hexagonal/rhombohedral stacking of graphite, and the mixing of diamond and graphite sequences within the structure (Figures S9-S11). Figure $4 \mathrm{~b}$ shows calculated diffraction patterns obtained by mixing different stacking-disordered graphite and cubic diamond sequences. The top diffraction pattern is that of stackingdisordered graphite showing that the feature at $\sim 12^{\circ} 2 \theta$ $\operatorname{MoK} \alpha$ remains sharp despite the disorder in the $\mathrm{sp}^{2}$-bonded layered material. Upon mixing into cubic diamond sequences in a random fashion, this feature is predicted to broaden and shifts toward higher angles as increasing amounts of diaphite sequences are created within the diamond lattice. The sharp peak at $\sim 12^{\circ} 2 \theta \mathrm{MoK} \alpha$ that is most clearly observed in the experimental data sets is thus thought to originate mainly from extended (potentially stacking disordered) graphitic regions included within the sample or from separate graphite inclusions. Diffraction intensity to the low-angle side of the main peak $\left(\sim 20^{\circ} 2 \theta \mathrm{MoK} \alpha\right)$ occurs for the cubic diamond containing even relatively small amounts of graphitic diaphite sequences. The appearance of this shoulder is typically interpreted as $c / h$ stacking-disordered diamond or "lonsdaleite" (Figure 4c). However, the diffraction intensity appearing on the high angle side of the main peak $\left(\sim 20^{\circ} 2 \theta \mathrm{MoK} \alpha\right)$ and that occurring at $\sim 34^{\circ} 2 \theta \mathrm{MoK} \alpha$ cannot be reproduced by mixing graphitic sequences with diamond, and so these features are likely to be characteristic of the $\mathrm{sp}^{3}$-bonded, $c / h$ stacking-disordered, structures. We conclude that although some contribution from type 1 diaphite nanostructures may be present in those samples that we and others have investigated to date, its quantification using X-ray diffraction is challenging. The type 1 diaphite structures observed in the HRTEM images of Figure 1 are only a few $\mathrm{nm}$ in width, which explains why they might be difficult to capture in X-ray diffraction studies of the bulk materials.

The implementation of type 2 diaphite into a DIFFaX model is difficult since the stacked layers consist of regions of $\mathrm{sp}^{3}$ - and $\mathrm{sp}^{2}$-bonded carbon. In Figure S12 the calculated diffraction pattern of a type 2 diaphite unit cell with $g=7$ and $d=7$ is shown. A characteristic feature of a type 2 diaphite is the quite short interlayer distance within the graphitic domains, which results in a Bragg peak at $\sim 13.4^{\circ} 2 \theta \mathrm{MoK} \alpha$ (corresponding to $\sim 3 \AA$ ). The absence of such a feature in our XRD data can be attributed to the overall low concentration of type 2 structures. Furthermore, due to the small domain sizes, any type 2 diaphite diffraction features would be expected to be very broad. 

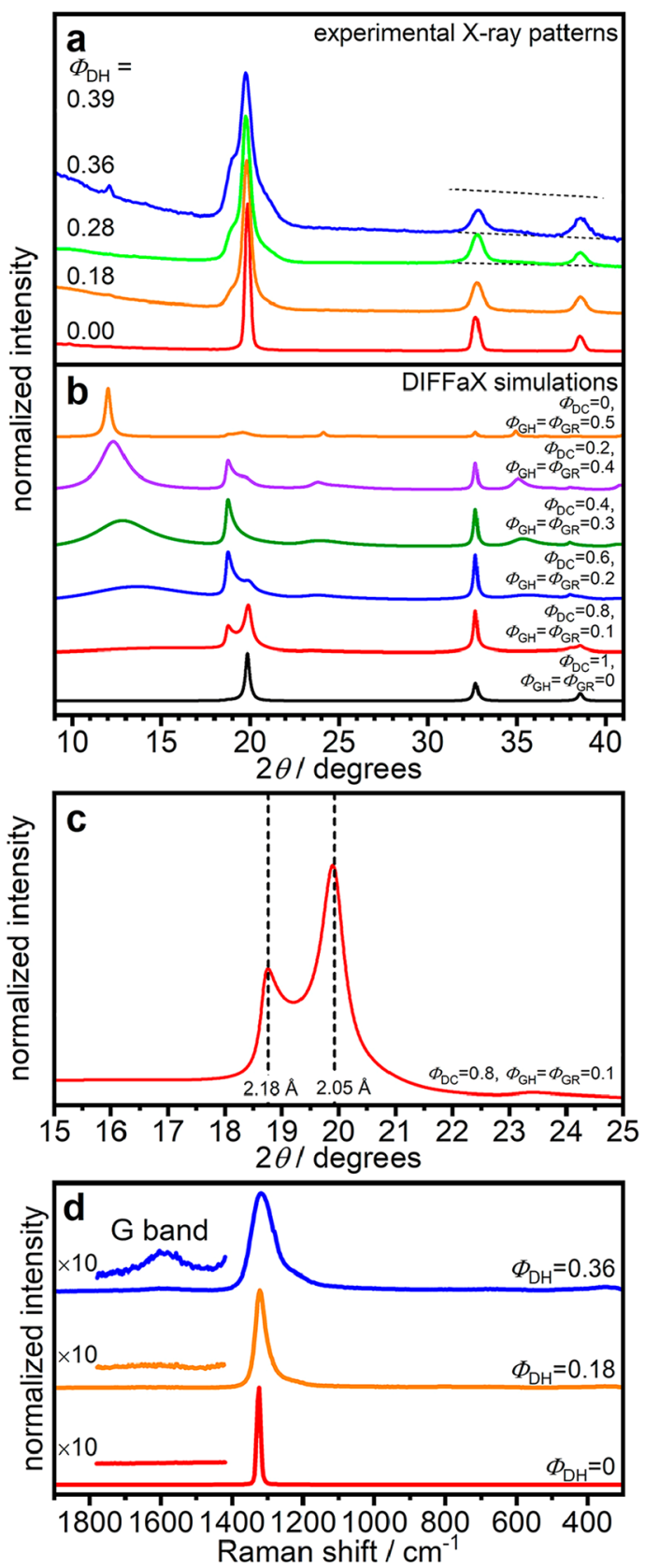

Figure 4. X-ray diffraction and Raman spectroscopy of Popigai diamonds. (a) Experimental X-ray diffraction patterns (MoK $\alpha: \lambda=$ $0.71073 \AA)$ with different hexagonality indices $\left(\Phi_{\mathrm{DH}}\right)$ as indicated in ref 17 . The dashed lines highlight a diffuse diffraction intensity around $35^{\circ}$. (b) Simulated X-ray diffraction patterns of stackingdisordered structures containing cubic diamond $\left(\Phi_{\mathrm{DC}}\right)$, diamond to graphite $\left(\Phi_{\mathrm{DG}}\right)$, graphite to diamond $\left(\Phi_{\mathrm{GD}}\right)$, and stacking-disordered graphite $\left(\Phi_{\mathrm{GH}}\right.$ and $\left.\Phi_{\mathrm{GR}}\right)$ sequences with random switching between diamond and graphite. The various stacking probabilities are given for each pattern, and the DIFFaX model developed for this study is described in more detail in the Supporting Information. (c) Simulated diffraction data in the angle range of the main diffraction feature show a doublet peak. (d) Microbeam Raman spectra (adapted with permission from ref 17, $514 \mathrm{~nm}$ excitation. Copyright 2019 Springer) of Popigai diamonds with different hexagonality indices. A weak feature due to the $\mathrm{G}$ band of graphitic structures contained within the sample is observed at $\sim 1600 \mathrm{~cm}^{-1}$ for the sample with $\Phi_{\mathrm{DH}}=0.36$. This is indicative of $\mathrm{sp}^{2}$ carbon associated with either flakes of a separate graphite phase or diaphite nanostructures.
The Raman spectrum for the sample with the highest hexagonality value reveals a broad and weak feature in the region of the $G$ band, which could imply the occurrence of diaphite domains within the material, although it could also be associated with a bulk disordered graphitic phase (Figure 4d). In summary, analysis of X-ray diffraction and Raman spectral profiles is most sensitive to determining the overall $c / h$ diamond stacking rather than revealing the existence of diaphite $\mathrm{sp}^{2}-\mathrm{sp}^{3}$ bonding within the samples.

The presence of diaphite nanostructures can help us to understand the large number of $\mathrm{sp}^{2}-\mathrm{sp}^{3}$-bonded carbon phases that are reported to form during the graphite to diamond transition, initiated by static or shock compression or during metastable syntheses from precursor compounds during chemical vapor deposition. Static compression of graphite at room $T$ suggests a sluggish transformation to a metastable phase at $\sim 19 \mathrm{GPa}$ : This material is optically transparent, exhibits superhard properties rivalling diamond, and returns to a mainly $\mathrm{sp}^{2}$-bonded material upon recovery. ${ }^{7,37-40}$ Proposed structure models for this material include "M-carbon", which according to Wang et al. ${ }^{39}$ contains only $\mathrm{sp}^{3}$-bonded carbon atoms. However, EELS data presented by Mao et al. ${ }^{40}$ suggest that cold compressed graphite transforms at $\sim 17 \mathrm{GPa}$ to a superhard phase with mixed $\mathrm{sp}^{2}$ - and $\mathrm{sp}^{3}$-bonded $\mathrm{C}$ atoms. Furthermore, a diffraction line that appears between the (100) and (101) peaks of graphite $(\sim 2.06 \AA)$, that was used to identify Mcarbon, ${ }^{40}$ is consistent with the diamond-type contribution from diaphite nanocomposite structures reported here (Figure $4 c)$. Formation of diaphite nanostructures during compression of graphite could provides an efficient pathway to initiate transformation to $\mathrm{sp}^{3}$-bonded phases such as diamond, lonsdaleite, and M-carbon.

Shock studies carried out along the principal Hugoniot of graphite to $100 \mathrm{GPa}$ show an inflection near $20 \mathrm{GPa}$ leading toward the pressure-density relations of $\mathrm{sp}^{3}$-bonded structures, although only very small quantities of crystalline diamond are recovered at ambient conditions. ${ }^{44}$ Laser shock experiments combined with in situ X-ray diffraction indicate formation of cubic diamond above $50 \mathrm{GPa}$ and of hexagonal diamond above $170 \mathrm{GPa}^{20}$ The evidence for the latter has been questioned as the doublet observed in the XRD patterns could be interpreted as arising from diamond structures experiencing different residual strain regimes. ${ }^{17}$ An alternative explanation for data presented in ref 20 could be formation of diaphite nanostructures during dynamic compression, for which diffraction data would also present a doublet peak (Figure 4c). A further study of graphite-diamond transformation using gas-gun shock experiments combined with synchrotron X-ray diffraction concluded that only elastically strained hexagonal diamond was produced above $50 \mathrm{GPa}^{21}$ The projection of these X-ray diffraction data most likely corresponds to that of type 2 diaphite shown in Figure 2. The data from these previous studies are consistent with formation of materials containing diaphite nanostructures, both within the compression phase or the rarefaction wave associated with shock studies, or during their recovery to ambient conditions.

Increased hardness and improved mechanical properties have been suggested for lonsdaleite diamond structures containing a high proportion of hexagonal stacking, ${ }^{45}$ consistent with results for cubic-hexagonal cBN/wBN polytype assemblies. ${ }^{19}$ Although our DFT calculations for bulk, shear, and Young's moduli of different type 1 and 2 
Table 1. Calculated Elastic and Mechanical Properties of 3C and 2H Diamonds and Type 2 Diaphite Structures Together with Experimental Data for 3C Diamond ${ }^{a}$

\begin{tabular}{lcccccc} 
& method & bulk modulus $(\mathrm{GPa})$ & shear modulus $(\mathrm{GPa})$ & Young's modulus (GPa) & Poisson ratio & Vickers hardness $(\mathrm{GPa})$ \\
\hline 3C diamond & experiment & $444^{46}$ & $535^{46}$ & $1143^{47}$ & $0.069^{47}$ & $79.7^{48}$ \\
& DFT (this work) & 454 & 558 & 1184 & 0.065 & 84.3 \\
2H diamond & & 453 & 562 & 1186 & 0.064 & 84.8 \\
$(g=1, d=1)$ & & 392 & 376 & 761 & 0.171 & 56.8 \\
$(g=1, d=3)$ & 402 & 407 & 838 & 0.149 & 61.5 \\
$(g=2, d=2)$ & 390 & 349 & 615 & 0.230 & 52.8 \\
$(g=3, d=1)$ & 379 & 300 & 395 & 0.321 & 45.7 \\
$(g=1, d=5)$ & 411 & 429 & 897 & 0.134 & 64.8
\end{tabular}

${ }^{a}$ Calculated Vickers hardness $\left(V_{\mathrm{H}}\right)$ values are determined using the relationship proposed by Teter ${ }^{49}\left(V_{\mathrm{H}}=0.151 G\right.$, where $G$ is the shear modulus).

diaphite models show lowered compressibility and tensile strength compared with fully $\mathrm{sp}^{3}$-bonded polymorphs (Table 1 ), we predict that inclusion of graphitic units within the nanocomposite diaphite nanostructures will result in improved fracture toughness caused by the lateral flexibility afforded by the $\mathrm{sp}^{2}$-bonded domains.

In conclusion, we provide evidence for the existence of novel type 1 and type 2 diamond-graphene nanocomposites within diamond materials recovered from natural impact materials and laboratory-shocked graphite. These nanostructures are characterized by the intimate association of $\mathrm{sp}^{3}$ (diamond) and $\mathrm{sp}^{2}$-bonded (graphene) domains, and the building units have a lateral width of a few $\mathrm{nm}$. Their diffraction signatures exhibit features that are consistent with those previously reported for lonsdaleite as well as the postgraphite "M-carbon" phase. The calculated $E(V)$ relationships reveal that these nanostructures provide low-energy solutions to structural transformation between fully $\mathrm{sp}^{2}$ - and $\mathrm{sp}^{3}$-bonded carbon allotropes that can be sampled under static and dynamic compression, including during the rarefaction wave associated with shock compression and during recovery to ambient conditions. The nanocomposite nature of the diaphite structures is expected to lead to mechanical behavior that preserves the superhard and incompressible properties of the $\mathrm{sp}^{3}$-bonded units, while leading to fracture toughening due to the angular flexibility and tensile resistance of the graphitic domains bonded to them.

\section{ASSOCIATED CONTENT}

\section{SI Supporting Information}

The Supporting Information is available free of charge at https://pubs.acs.org/doi/10.1021/acs.nanolett.0c00556.

Details of experimental methods include samples; transmission electron microscopy, image processing and image simulation as well as Figures S1-S3; computational methods and Figures S4-S8; DIFFaX modeling and Figures S9-S11 as well as Tables S1-S3; Figure S12; and supplementary references (PDF)

\section{AUTHOR INFORMATION}

\section{Corresponding Authors}

Péter Németh - Institute of Materials and Environmental Chemistry, Research Centre for Natural Sciences, 1117 Budapest, Hungary; Department of Earth and Environmental Sciences, University of Pannonia, 8200 Veszprem, Hungary; ำ orcid.org/0000-0001-5592-5877; Email: nemeth.peter@ ttk.mta.hu
Kit McColl - Department of Chemistry, University College London, London WC1H OAJ, United Kingdom; 이이.org/ 0000-0002-7794-8276; Email: kit.mccoll.10@ucl.ac.uk

Christoph G. Salzmann - Department of Chemistry, University College London, London WC1H OAJ, United Kingdom; 이이.org/0000-0002-0714-7342; Email: c.salzmann@ucl.ac.uk

Paul F. McMillan - Department of Chemistry, University College London, London WC1H OAJ, United Kingdom; ○ orcid.org/0000-0002-5298-780X; Email: p.f.mcmillan@ ucl.ac.uk

\section{Authors}

Rachael L. Smith - Department of Chemistry, University College London, London WC1H OAJ, United Kingdom

Mara Murri - Department of Earth and Environmental Sciences, University of Pavia, 27100 Pavia, Italy; Department of Earth and Environmental Sciences, University of MilanoBicocca, I-20126 Milano, Italy

Laurence A. J. Garvie - Center for Meteorite Studies, Arizona State University, Tempe, Arizona 85287-6004, United States

Matteo Alvaro - Department of Earth and Environmental Sciences, University of Pavia, 27100 Pavia, Italy

Béla Pécz - Institute of Technical Physics and Materials Science, Centre for Energy Research, 1121 Budapest, Hungary

Adrian P. Jones - Department of Earth Sciences, University College London, WC1E 6BT London, United Kingdom

Furio Corà - Department of Chemistry, University College London, London WC1H OAJ, United Kingdom

Complete contact information is available at:

https://pubs.acs.org/10.1021/acs.nanolett.0c00556

\section{Author Contributions}

These authors contributed equally. P.N., K.M., P.F.M., and C.G.S. initiated and coordinated different aspects of the project. A.J. and L.A.J.G. provided some of the samples and carried out the initial characterization. B.P., L.A.J.G., and P.N. were involved in the HRTEM investigation, and the data were interpreted by L.A.J.G. and P.N. K.M. and F.C. carried out DFT calculations and modeling. M.M. and M.A. obtained the X-ray data. R.L.S. and C.G.S. carried out the DIFFAX modeling. P.F.M., C.G.S., K.M., P.N., and L.A.J.G. collaborated on writing the manuscript, and figures were prepared by P.N., K.M., and R.L.S. All authors participated in discussion of the results and have given approval to the final version of the manuscript.

\section{Notes}

The authors declare no competing financial interest. 


\section{ACKNOWLEDGMENTS}

We are grateful to the staff and for use of the facilities in the John M. Cowley Center for High Resolution Electron Microscopy at Arizona State University, the Titan HRTEM facility located in the School of Mines at Imperial College London, and the Themis Titan HRTEM facility located at the Institute of Technical Physics and Materials Science, Centre for Energy Research. P.N. acknowledges financial support from the Hungarian National Research, Development and Innovation Office project NKFIH_KH126502, the János Bolyai Research Scholarship, and ÚNKP-19-4-PE-4 New National Excellence Program of the Ministry for Innovation and Technology. L.A.J.G. was supported by a NASA Emerging Worlds grant NNX17AE56G. B.P. thanks the support of the project VEKOP-2.3.3-15-2016-00002. R.L.S. thanks the UCL Chemistry Department for a DTP studentship. C.G.S. has received funding from the European Research Council under the European Union's Horizon 2020 research and innovation program (grant agreement no. 725271). This work made use of the ARCHER UK National Supercomputing Service (http://www.archer.ac.uk) via K.M. and F.C.'s membership of the UK's HEC Materials Chemistry Consortium, which is funded by EPSRC (EP/L000202). K.M. and F.C. gratefully acknowledge HPC resources provided by the UK Materials and Molecular Modelling Hub, which is partially funded by EPSRC (EP/P020194/1), and UCL Grace and Kathleen HPC Facilities and associated support services, in completion of this work. M.M. and M.A. were supported by the IMPACt (R164WEJAHH) and the TRUE DEPTHS (ERC grant 714936) projects. M.M. also received support from the Barringer Family Fund for Meteorite Impact Research and the EU Horizon 2020 research and innovation program as Europlanet 2020 RI (no. 654208).

\section{REFERENCES}

(1) Hazen, R. M.; Jones, A. P.; Baross, J. A. Reviews in Mineralogy and Geochemistry. Carbon in Earth; Mineralogical Society of America, Geochemical Society: Washington, DC, 2013.

(2) Langenhorst, F.; Campione, M. Ideal and real structures of carbon forms with some remarks on the geological significance. $J$. Geol. Soc. 2019, 176, 337-347.

(3) Ferrari, A. C.; et al. Science and technology roadmap for graphene, related two-dimensional crystals, and hybrid systems. Nanoscale 2015, 7, 4598-4810.

(4) Frondel, C.; Marvin, U. B. Lonsdaleite, a hexagonal polymorph of diamond. Nature 1967, 214, 587-589.

(5) Hanneman, R. E.; Strong, H. M.; Bundy, F. P. Hexagonal diamonds in meteorites: Implications. Science 1967, 155, 995-997.

(6) Bundy, F. P.; Kasper, J. S. Hexagonal diamond - A new form of carbon. J. Chem. Phys. 1967, 46, 3437-3446.

(7) Yagi, T.; Utsumi, W.; Yamakata, M.; Kikegawa, T.; Shimomura, O. High-pressure in situ X-ray-diffraction study of the phase transformation from graphite to hexagonal diamond at room temperature. Phys. Rev. B: Condens. Matter Mater. Phys. 1992, 46, 6031.

(8) Erskine, D. J.; Nellis, W. J. Shock-induced martensitic phase transformation of oriented graphite to diamond. Nature 1991, 349, 317-319.

(9) Le Guillou, C.; Rouzaud, J. N.; Remusat, L.; Jambon, A.; Bourot-Denise, M. Structures, origin and evolution of various carbon phases in the ureilite Northwest Africa 4742 compared with laboratory-shocked graphite. Geochim. Cosmochim. Acta 2010, 74 (14), 4167-4185.

(10) Isobe, F.; Ohfuji, H.; Sumiya, H.; Irifune, T. Nanolayered diamond sintered compact obtained by direct conversion from highly oriented graphite under high pressure and high temperature. J. Nanomater. 2013, 2013, 380165.

(11) Kvasnytsya, V.; et al. New evidence of meteoritic origin of the Tunguska cosmic body. Planet. Space Sci. 2013, 84, 131-140.

(12) Kulnitskiy, B.; Perezhogin, I.; Dubitsky, G.; Blank, V. Polytypes and twins in the diamond-lonsdaleite system formed by high-pressure and high-temperature treatment of graphite. Acta Crystallogr., Sect. B: Struct. Sci., Cryst. Eng. Mater. 2013, 69, 474-479.

(13) Ohfuji, H.; et al. Natural occurrence of pure nanopolycrystalline diamond from impact crater. Sci. Rep. 2015, 5, 14702.

(14) Németh, P.; et al. Lonsdaleite is faulted and twinned cubic diamond and does not exist as a discrete material. Nat. Commun. 2014, 5, 6447.

(15) Németh, P.; Garvie, L. A. J.; Buseck, P. R. Twinning of cubic diamond explains reported nanodiamond polymorphs. Sci. Rep. 2016, 5, 18381 .

(16) Salzmann, C. G.; Murray, B. J.; Shephard, J. J. Extent of stacking disorder in diamond. Diamond Relat. Mater. 2015, 59, 6972 .

(17) Murri, M.; et al. Quantifying hexagonal stacking in diamond. Sci. Rep. 2019, 9, 10334.

(18) Belenkov, E. A.; Greshnyakov, V. A. Structure Formation of Hexagonal Diamond: Ab Initio Calculations. Phys. Solid State 2019, 61 (10), 1882-1890.

(19) Pan, Z.; Sun, H.; Zhang, Y.; Chen, C. Harder than diamond: superior indentation strength of wurtzite $\mathrm{BN}$ and lonsdaleite. Phys. Rev. Lett. 2009, 102, No. 055503.

(20) Kraus, D.; et al. Nanosecond formation of diamond and lonsdaleite by shock compression of graphite. Nat. Commun. 2016, 7, 10970.

(21) Turneaure, S. J.; Sharma, S. M.; Volz, T. J.; Winey, J. M.; Gupta, Y. M. Transformation of shock-compressed graphite to hexagonal diamond in nanoseconds. Sci. Adv. 2017, 3, eaao3561.

(22) Boulfelfel, A. E.; Oganov, A. R.; Leoni, S. Understanding the nature of "superhard graphite". Sci. Rep. 2012, 2, 471.

(23) Hoffmann, R.; Kabanov, A. A.; Golov, A. A.; Proserpio, D. M. Homo Citans and Carbon Allotropes: For an Ethics of Citation. Angew. Chem., Int. Ed. 2016, 55, 10962-10976.

(24) Avery, P.; et al. Predicting superhard materials via a machine learning informed evolutionary structure search. npj Comput. Mater. 2019, 5, 89.

(25) Mundy, C. J.; et al. Ultrafast transformation of graphite to diamond: an ab initio study of graphite under shock compression. J. Chem. Phys. 2008, 128 (18), 184701.

(26) Khaliullin, R. Z.; Eshet, H.; Kühne, T. D.; Behler, J.; Parrinello, M. Nucleation mechanism for the direct graphite-to-diamond phase transition. Nat. Mater. 2011, 10, 693-697.

(27) Garvie, L. A. J.; Németh, P.; Buseck, P. R. Transformation of graphite to diamond via a topotactic mechanism. Am. Mineral. 2014, 99 (2-3), 531-538.

(28) Kis, V. K.; Shumilova, T.; Masaitis, V. HRTEM study of Popigai impact diamond: heterogeneous diamond nanostructures in native amorphous carbon matrix. Phys. Chem. Miner. 2016, 43, 661670.

(29) Németh, P.; Garvie, L. A. J. Extraterrestrial, shock-formed, cage-like nanostructured carbonaceous materials. Am. Mineral. 2020, 105 (2), 276-281.

(30) Regan, B.; et al. Plastic Deformation of Single-Crystal Diamond Nanopillars. Adv. Mater. 2020, 32, 1906458.

(31) Jones, A. P.; et al. Structural characterization of natural diamond shocked to $60 \mathrm{GPa}$; implications for Earth and planetary systems. Lithos 2016, 265, 214-221.

(32) Chu, P. K.; Li, L. Characterization of amorphous and nanocrystalline carbon films. Mater. Chem. Phys. 2006, 96 (2-3), 253-277.

(33) Robertson, J. Diamond-like amorphous carbon. Mater. Sci. Eng., $R$ 2002, 37 (4-6), 129-281. 
(34) Hasegawa, G.; et al. Studies on electrochemical sodium storage into hard carbons with binder-free monolithic electrodes. ChemElectroChem 2015, 2, 1917.

(35) Ohnishi, H.; Nasu, K. Generation and growth of $\mathrm{sp}^{3}$-bonded domains by visible photon irradiation of graphite. Phys. Rev. B: Condens. Matter Mater. Phys. 2009, 80, No. 014112.

(36) Kanasaki, J.; Inami, E.; Tanimura, K.; Ohnishi, H.; Nasu, K. Formation of $\mathrm{sp}^{3}$-Bonded Carbon Nanostructures by Femtosecond Laser Excitation of Graphite. Phys. Rev. Lett. 2009, 102, No. 087402.

(37) Miller, E. D.; Nesting, D. C.; Badding, J. V. Quenchable Transparent Phase of Carbon. Chem. Mater. 1997, 9, 18-22.

(38) Amsler, M.; et al. Crystal Structure of Cold Compressed Graphite. Phys. Rev. Lett. 2012, 108, No. 065501.

(39) Wang, Y.; Panzik, J. E.; Kiefer, B.; Lee, K. K. M. Crystal structure of graphite under room-temperature compression and decompression. Sci. Rep. 2012, 2, 520.

(40) Mao, W. L.; et al. Bonding changes in compressed superhard graphite. Science 2003, 302 (5644), 425-427.

(41) Gogotsi, G. A. Fracture toughness of ceramics and ceramic composites. Ceram. Int. 2003, 29, 777-784.

(42) Pandey, K. C. New dimerized-chain model for the reconstruction of the diamond $(111)-(2 \times 1)$ surface. Phys. Rev. B: Condens. Matter Mater. Phys. 1982, 25, 4338.

(43) De Vita, A.; Galli, G.; Canning, A.; Car, R. A microscopic model for surface-induced diamond-to-graphite transitions. Nature 1996, 379, 523-526.

(44) Wlodarczyk, E.; Trebinski, R. An interpretation of the shock Hugoniot of graphite. J. Phys. IV 1991, C3, 533-538.

(45) Baek, W.; et al. Unique Nanomechanical Properties of Diamond-Lonsdaleite Biphases: Combined Experimental and Theoretical Consideration of Popigai Impact Diamonds. Nano Lett. 2019, 19 (3), 1570-1576.

(46) Angel, R. J.; Alvaro, M.; Nestola, F.; Mazzucchelli, M. L. Diamond thermoelastic properties and implications for determining the pressure of formation of diamond-inclusion systems. Russ. Geol. Geophys. 2015, 56, 211-220.

(47) Klein, C. A.; Cardinale, G. F. Young's modulus and poisson's ratio of CVD diamond. Diamond Relat. Mater. 1993, 2 (5-7), 918923.

(48) Dub, S.; et al. Vickers hardness of diamond and cBN single crystals: AFM approach. Crystals 2017, 7 (12), 369.

(49) Teter, D. M. Computational Alchemy: The Search for New Superhard Materials. MRS Bull. 1998, 23, 22-27. 